\title{
Criminologie
}

\section{La trajectoire de la recherche empirique quantitative dans la revue Criminologie (1968-2016) : une perspective éditoriale Empirical quantitative research trajectory in Criminologie journal (1968-2016): An editorial perspective La trayectoria de la investigación empírica cuantitativa en la revista Criminologie (1968-2016): Una perspectiva editorial}

\author{
Yanick Charette, Pierre Tremblay et Nadine Deslauriers-Varin
}

Volume 51, numéro 1, printemps 2018

50 ans de Criminologie

URI : https://id.erudit.org/iderudit/1045312ar

DOI : https://doi.org/10.7202/1045312ar

Aller au sommaire du numéro

\section{Éditeur(s)}

Les Presses de l’Université de Montréal

ISSN

0316-0041 (imprimé)

1492-1367 (numérique)

Découvrir la revue

Citer cet article

Charette, Y., Tremblay, P. \& Deslauriers-Varin, N. (2018). La trajectoire de la recherche empirique quantitative dans la revue Criminologie (1968-2016) : une perspective éditoriale. Criminologie, 51(1), 169-199.

https://doi.org/10.7202/1045312ar

\section{Résumé de l'article}

Chaque numéro publié d'une revue scientifique vient clore plusieurs rondes de tractations qui débutent au sein du comité de rédaction, se déplacent ensuite autour de ceux qui ont accepté de participer au numéro et se ramifient et se prolongent durant les évaluations et les révisions des manuscrits qui seront finalement publiés. Les directeurs successifs sont contraints de balancer la part relative des articles de réflexion et des articles d'investigation, la proportion relative des enquêtes empiriques qui procèdent par vérification d'hypothèses et celle des investigations ouvertes et exploratoires. En se basant sur l'ensemble des articles publiés dans la revue Criminologie de 1968 à 2016 ( $n=$ 658), cette étude s'intéresse à l'effet des différentes stratégies éditoriales mises en place sur la part relative qui sera accordée à la recherche empirique quantitative dans le discours criminologique. Les analyses montrent que les directeurs, par l'intermédiaire des comités de rédaction, peuvent agir sur l'offre par des innovations éditoriales (introduction de numéros thématiques, publication semestrielle, ouverture aux publications hors thèmes, diversification de la provenance des auteurs), mais aussi contrôler cette offre par une hausse des standards (uniformité de la taille des articles, complexité des analyses) et par une sollicitation active d'articles innovateurs. Ces innovations éditoriales ont diversifié le discours scientifique de la revue et ont assuré, au final, la survie de la revue durant un demi-siècle.
Tous droits réservés C Les Presses de l’Université de Montréal, 2018
Ce document est protégé par la loi sur le droit d'auteur. L’utilisation des services d'Érudit (y compris la reproduction) est assujettie à sa politique d'utilisation que vous pouvez consulter en ligne.

https://apropos.erudit.org/fr/usagers/politique-dutilisation/ 


\title{
La trajectoire de la recherche empirique quantitative dans la revue Criminologie (1968-2016) : une perspective éditoriale
}

\author{
Yanick Charette ${ }^{1}$ \\ Professeur adjoint \\ École de travail social et de criminologie, Université Laval \\ yanick.charette.1@ulaval.ca \\ Pierre Tremblay \\ Professeur titulaire \\ École de criminologie, Université de Montréal \\ pierre.tremblay@umontreal.ca \\ Nadine Deslauriers-Varin \\ Professeure agrégée \\ École de travail social et de criminologie, Université Laval \\ nadine.deslauriers-varin@tsc.ulaval.ca
}

RÉSUMÉ - Chaque numéro publié d'une revue scientifique vient clore plusieurs rondes de tractations qui débutent au sein du comité de rédaction, se déplacent ensuite autour de ceux qui ont accepté de participer au numéro et se ramifient et se prolongent durant les évaluations et les révisions des manuscrits qui seront finalement publiés. Les directeurs successifs sont contraints de balancer la part relative des articles de réflexion et des articles d'investigation, la proportion relative des enquêtes empiriques qui procèdent par vérification d'hypothèses et celle des investigations ouvertes et exploratoires. En se basant sur l'ensemble des articles publiés dans la revue Criminologie de 1968 à $2016(\mathrm{n}=658)$, cette étude s'intéresse à l'effet des différentes stratégies éditoriales mises en place sur la part relative qui sera accordée à la recherche empirique quantitative dans le discours criminologique. Les analyses montrent que les directeurs, par l'intermédiaire des comités de rédaction, peuvent agir sur l'offre par des innovations éditoriales (introduction de numéros thématiques, publication semestrielle, ouverture aux publications hors thèmes, diversification de la provenance des auteurs), mais aussi

1. École de travail social et de criminologie, Université Laval, Pavillon Charles-De Koninck, 1030, av. des Sciences-Humaines, Local 5444, Québec (Québec), Canada, G1V 0A6.

Criminologie, vol. 51, $\mathrm{n}^{\circ} 1$ (2018) 
contrôler cette offre par une hausse des standards (uniformité de la taille des articles, complexité des analyses) et par une sollicitation active d'articles innovateurs. Ces innovations éditoriales ont diversifié le discours scientifique de la revue et ont assuré, au final, la survie de la revue durant un demi-siècle.

MotS CLÉS - Revue Criminologie, méthode quantitative, innovations, processus éditorial, analyse historique.

\section{Introduction}

Les revues scientifiques ont joué un rôle majeur dans le processus d'institutionnalisation des sciences sociales au Québec. Dans le cas de la criminologie, Poupart (2004) suggère que la création d'une revue spécialisée (Acta Criminologica depuis 1968, Criminologie depuis 1975) a contribué à l'autonomie de la criminologie comme discipline universitaire, à la professionnalisation de ses diplômés et aussi à sa légitimité institutionnelle auprès des agences gouvernementales à travers les organismes subventionnaires qui financent les recherches qui y sont publiées.

L'existence de la revue Criminologie, après un demi-siècle, semble aller de soi. Mais elle ne l'était pas à ses débuts. Pourquoi créer une revue spécialisée pour des articles qui auraient pu tout aussi bien être publiés dans d'autres revues scientifiques mieux établies et rattachées historiquement à ses disciplines mères de la criminologie (psychologie, sociologie, psychiatrie principalement)? Rien ne garantissait non plus qu'une fois créée, cette revue parvienne à survivre aux incertitudes propres à une telle entreprise. Il n'est pas inutile de rappeler que chaque volume publié (deux par année depuis 1977) vient clore plusieurs rondes de tractations qui débutent au sein du comité de rédaction, se déplacent ensuite autour de ceux qui ont accepté de participer au numéro et se ramifient et se prolongent durant les évaluations et les révisions des manuscrits qui seront finalement publiés. On peut diverger d'opinion sur la thématique, le type de recherche que l'on devrait privilégier ou la part accordée aux synthèses conceptuelles et les standards de rigueur qui doivent être imposés aux auteurs qui soumettent leurs manuscrits, et ce, de manière équitable pour s'assurer de l'absence de traitement différentiel. S'il n'existe pas une volonté commune de privilégier le compromis, l'ouverture et la tolérance, les chances de survie d'une revue scientifique sont minces. Et bien entendu, comme c'est le cas pour toute entreprise collective, c'est le directeur de la revue qui, en fin de compte, sera tenu responsable de l'issue heureuse ou malheureuse des tractations qui se sont faites sous sa direction, même s'il n'y est pas directement impliqué. 
Dans cette étude, nous nous intéressons à la part relative qu'occupe la recherche empirique quantitative dans le discours criminologique que présente la revue Criminologie de 1968 à 2016. Comme le soulignait Poupart (2004), les directeurs de cette revue opèrent dans un contexte très particulier:

dans la mesure où ce n'est pas partout que l'on retrouve une discipline spécifiquement identifiée à la criminologie, et que ce n'est pas non plus partout qu'il existe sur le marché du travail une catégorie de professionnels clairement identifiée à cette discipline: le criminologue. (p. 72)

On peut donc penser ou espérer que les directeurs successifs de la revue sont contraints d'équilibrer la part relative des articles de réflexion et des articles d'investigation, la proportion relative des enquêtes empiriques qui procèdent par vérification d'hypothèses et celle des investigations ouvertes et exploratoires. Les revues scientifiques sont soumises, comme tout marché, aux règles de l'offre (un espace de publication qui requiert des exigences que les auteurs doivent satisfaire en échange du capital symbolique que procure le prestige de la publication) et la demande (les auteurs qui désirent utiliser cet espace en lui soumettant des manuscrits). Les directeurs, par l'intermédiaire des comités de rédaction dont ils s'entourent ou des évaluateurs qu'ils recrutent, peuvent, dans une certaine mesure, agir sur l'offre par des innovations éditoriales, mais aussi contrôler cette offre par une hausse des standards et par une sollicitation active d'articles innovateurs. En d'autres mots: comment les directeurs de la revue ont-ils contribué à préserver son existence pendant une aussi longue période?

\section{Données}

Le corpus de cette étude est composé des textes publiés dans la revue Criminologie. Ceux-ci furent lus et analysés depuis sa création, en 1968, jusqu'en $2016(n=811)$. Un certain nombre de ces textes $(n=153$ textes; $19 \%$ ) ont été retirés parce qu'ils ne se qualifiaient pas comme des études distinctes (ex. : éditoriaux, bibliographies, introductions aux numéros spéciaux). Les documents retenus comme des articles $(n=658)$ furent classés en deux catégories: (1) les articles empiriques qui avaient construit explicitement un corpus original de données et qui avaient appuyé leurs analyses en s'inspirant d'autres études empiriques ou théoriques apparentées $(n=305 ; 46 \%)$ et (2) les articles non empiriques 
qui développent une argumentation réflexive en s'appuyant sur un corpus pertinent d'études apparentées $(n=353 ; 54 \%)$. Les articles empiriques ont été à leur tour classés en deux catégories: ceux qui présentent un ensemble défini d'observations, d'entretiens libres ou semi-structurés ou d'une documentation relative à un objet factuel d'étude ont été définis comme des articles qualitatifs ( $n=120 ; 39 \%$ des articles empiriques), alors que les études dont les observations ou les entretiens font l'objet d'une analyse statistique ont été définies comme des études quantitatives ( $n=185 ; 61 \%$ des articles empiriques).

Depuis un demi-siècle, sept directeurs de la revue se sont succédé: Denis Szabo (1968-1986; $n=178 ; 28 \%)$, André Normandeau (1987$1988 ; n=22 ; 3 \%)$, Serge Brochu (1989-1997; $n=110 ; 17 \%)$, Pierre Landreville (1997-2005; $n=113 ; 18 \%)$, Dianne Casoni (2005-2012; $n=140,22 \%)$, Jo-Anne Wemmers (2013-2015; $n=71 ; 11 \%)$, Chloé Leclerc $(2016 ; n=11 ; 2 \%)$. Pour fins d'analyse, on a procédé au découpage de cinq périodes d'environ dix ans. La période des débuts (1968-1974) correspond aux années pendant lesquelles la revue (Acta Criminologica) était rattachée à un département plutôt qu'à une école professionnelle. Durant cette période, on retrouvait en moyenne 3,7 articles par numéro $(\sigma=0,8)$. La seconde période, de transition, correspond à la période où le rythme de parution des publications de la revue devient, en 1977, semestriel plutôt qu'annuel, et la revue est passée à 6,9 articles par numéro $(\sigma=2,5)$. Les deux périodes suivantes sont principalement définies par leur directeur et ont évolué parallèlement à la croissance des effectifs du corps professoral de l'École de criminologie et de ses diplômés de deuxième et troisième cycle (Poupart, 2004). La troisième période (1987-1996) incorpore la brève direction assurée par Normandeau ( 2 ans seulement) à celle de Brochu (9 ans), période durant laquelle 6,3 articles étaient publiés en moyenne $(\sigma=1,1)$. La quatrième période (1997-2004), soit celle sous la direction de Landreville, présentait en moyenne 7,1 articles $(\sigma=1,1)$. La cinquième période (2005-2016), incluant la brève direction assurée par Wemmers ( 3 ans) et par Leclerc ( 1 an seulement parce qu'elle est censurée à droite par la période d'observation) à celle de Casoni (7 ans), est celle où l'on a observé une augmentation notable du nombre d'articles par numéro, soit 10,2 en moyenne $(\sigma=3,1)$.

Plusieurs changements notables ayant influencé la revue et son contenu sont présents au cours des différentes périodes. Il ne serait pas étonnant que les sujets ayant retenu l'attention des auteurs de la revue 
aient aussi changé et que certains d'entre eux soient plus inspirants et que d'autres aient perdu de leur attraction. Les thèmes abordés dans la revue pourraient être classés en trois grandes catégories: les articles discutant des agents pénaux ( $n=286,44 \%$; p. ex.: tribunaux, $n=94$, $14 \%$; prison, $n=87,13 \%$; sécurité et police, $n=69,11 \%$ ), du délinquant et de ses délits $(n=253,38 \%$; p. ex.: délinquants juvéniles, $n=106,16 \%$; crime ou criminalité, $n=94,14 \%$; délinquants adultes, $n=71,11 \%)$ ou de thèmes résiduels $(n=182,28 \%$; p. ex.: victimes, $n=73,11 \%$; interventions, $n=64,10 \%$; femmes, $n=59,9 \%)^{2}$. Ces catégories ne sont pas mutuellement exclusives, un article pouvant combiner plusieurs thèmes à la fois. Toutefois, la majorité $(83 \%)$ des articles ne discute que d'un thème.

Outre ces changements structuraux ou reliés au développement de la criminologie, les directeurs de la revue ont aussi eu leur rôle à jouer. À cet effet, il est possible de constater que les directeurs ont introduit trois innovations éditoriales techniques au cours des différentes périodes à l'étude: l'introduction de numéros thématiques (1975), la publication de numéros semestriels plutôt qu'une livraison annuelle (1977) et l'ouverture aux publications hors thèmes (1989). Le format de la revue que nous connaissons aujourd'hui, en l'occurrence un numéro thématique accompagné d'une section ouverte composée d'articles hors thème, date de 1989.

Bien qu'elle ne soit pas ponctuelle comme les précédentes, on remarque une quatrième innovation éditoriale qui s'est développée au fil du temps: la diversité de la provenance des affiliations des auteurs. Nous considérons que la diffusion d'auteurs rattachés à des universités d'autres pays ou d'autres provinces canadiennes témoignerait d'une volonté des directeurs, et de leurs comités de rédaction, de diversifier le discours criminologique scientifique véhiculé dans la revue. À cet effet, notons que $41 \%$ des articles publiés par la revue ont été rédigés par des auteurs rattachés à l'École de criminologie de Montréal $(n=269)$, ce pourcentage grimpant à $66 \%$ lorsqu'on y rattache les auteurs affiliés à d'autres universités du Québec $(n=114)$ ou à d'autres départements de l'Université de Montréal $(n=49)$. En revanche, $23 \%$ des auteurs de la revue sont affiliés à des universités d'autres pays $(n=149)$ et $12 \%$ à des universités canadiennes situées dans d'autres provinces $(n=77)$.

2. Cette catégorisation par année a été offerte aux auteurs qui participent à ce numéro spécial. On trouvera ailleurs les critères qui ont été utilisés pour l'établir. 
L'influence de la provenance des auteurs fut donc aussi prise en considération dans le présent article. Si l'article était le produit de plusieurs auteurs, la provenance du premier auteur fut considérée.

Les directeurs doivent aussi assurer un contrôle de qualité. Ce contrôle qui s'exerce de différentes manières fut ici défini à l'aide de trois indicateurs. Le premier est celui de la variabilité de la longueur des articles qui constitue un indicateur de la volonté d'imposer les mêmes standards à tous les auteurs publiés. Les deux autres indicateurs de contrôles éditoriaux se limitent à notre objet d'étude, soit la recherche empirique quantitative dans les articles de la revue. Ces indicateurs pourraient traduire une volonté de hausser, de manière générale, les standards et la qualité des articles publiés, qu'ils soient empiriques ou non, quantitatifs ou non.

Le deuxième indicateur est celui de la complexité relative des analyses statistiques présentées dans les articles empiriques de nature quantitative. Ces articles sont classés en trois rangs: analyses univariées (moyenne, pourcentage; $n=59 ; 32 \%$ ), analyses bivariées seulement (tableau croisé, corrélation; $n=55 ; 30 \%$ ), analyses multivariables ${ }^{3}$ (régression multiple, analyse factorielle, analyses multiniveaux; $n=71$; $38 \%)$. Cette catégorisation est mutuellement exclusive et lorsque plus d'un type d'analyse était présenté, le plus complexe fut noté. Une baisse des articles empiriques purement descriptifs ou une hausse des analyses plus sophistiquées serait un indicateur d'une volonté d'élever le niveau de rigueur des articles.

Le troisième indicateur de contrôle éditorial, visant à hausser la qualité des articles, serait la fréquence des articles innovateurs publiés dans la revue. Le statut d'innovateur accordé à un article prend comme cadre de référence les articles publiés dans la revue Criminologie, peu importe que l'innovation d'origine puisse être attribuée à d'autres auteurs ayant publié dans d'autres revues. Ces innovations furent identifiées en feuilletant l'ensemble des méthodes utilisées par les articles quantitatifs dans la période d'observation et en notant celles qui se distinguaient par leur originalité. Pour fins d'analyse, les analyses univariées et bivariées étant tenues pour acquises, les innovations dites formelles, ou statistiques, renvoient aux auteurs qui ont présenté des techniques ou des modèles d'analyses multivariables qui n'avaient jamais

3. Les analyses multivariables sont souvent confondues avec les analyses multivariées, ces dernières incluant plus d'une variable dépendante, ce qui n'est pas automatiquement le cas des premières (Hidalgo et Goodman, 2013). 
été utilisés dans la revue (p. ex.: régression multiple, analyse multiniveau). Les innovations dites substantives renvoient aux articles (ou aux auteurs) qui introduisent dans l'histoire de la revue des corpus empiriques ou des données non traditionnelles (p. ex.: les sondages de délinquance autorévélée, les sondages de victimisation), des mesures originales (p. ex.: revenus criminels, capital social) ou des devis de recherche novateurs (p. ex.: devis expérimentaux ou quasi expérimentaux).

Les directeurs et leur comité éditorial prennent des décisions, mettent en place des structures de contrôle et des innovations qui vont façonner l'image de la revue. Il s'agira donc ici d'examiner l'influence de ces divers aspects structuraux ou éditoriaux quant à la place prise par les articles empiriques quantitatifs dans la revue Criminologie.

\section{Équilibrer les perspectives}

On ne peut exclure d'entrée de jeu que les directeurs de la revue Criminologie aient divergé d'opinion sur la manière de concevoir le discours criminologique. Si c'était le cas, on devrait s'attendre à des variations significatives dans la proportion relative des articles empiriques ou la place octroyée aux enquêtes empiriques quantitatives. La Figure 1 présente l'évolution de la part relative des articles de réflexion, d'investigation empirique qualitative et d'investigation empirique quantitative, dans Criminologie de 1968 à 2016 ${ }^{4}$. À première vue, il semble qu'au fil du temps les directeurs aient accordé une place plus importante à la criminologie empirique $(33 \%$ durant la période de transition, $44 \%$ sous la direction de Serge Brochu, $49 \%$ sous celle de Pierre Landreville, et $56 \%$ dans la dernière décennie). Par ailleurs, un changement appréciable se produit en 2005: un déclin marqué des recherches empiriques quantitatives. Les premiers directeurs, Denis Szabo, Serge Brochu et Pierre Landreville, présentaient en moyenne $67 \%, 81 \%$ et $78 \%$ d'articles empiriques quantitatifs, alors que parmi

4. La Figure 1 présente la proportion d'articles empiriques et d'articles empiriques utilisant une méthode quantitative estimée à l'aide d'une équation de régression logistique prenant l'année de publication comme prédicteur. Pour observer les tendances temporelles non linéaires, les fonctions polynomiales de l'année ont été extraites. Pour la prédiction de la tendance des articles empiriques, la fonction d'ordre cinq permettait un meilleur ajustement, alors que pour prédire la tendance des articles utilisant une méthode quantitative, la fonction d'ordre quatre fut privilégiée (voir Annexe, Modèles 1 et 5). Toutes les analyses de cet article ont été réalisées avec le logiciel en libre accès $R$ ( $R$ Development Core Team, 2017). 


\section{F I G U RE 1}

\section{Évolution des articles empiriques et quantitatifs publiés dans Criminologie (1968-2016)}

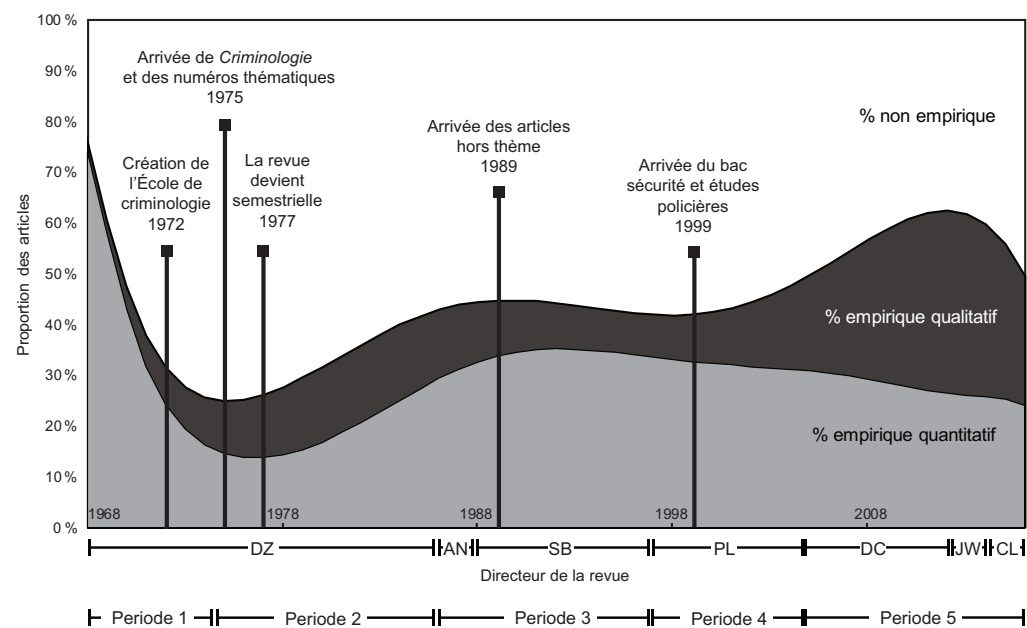

DZ : Denis Szabo; AN : André Normandeau; SB : Serge Brochu; PL : Pierre Landreville; DC : Dianne Casoni; JW : Jo-Anne Wemmers; CL : Chloé Leclerc

Note: Les courbes de tendance présentées dans cette figure sont extraites des modèles de régression logistique présentés en Annexe (Modèles 1,5).

les derniers, Dianne Casoni et Jo-Anne Wemmers, en présentaient en moyenne $43 \%$ et $49 \%$ respectivement 5 .

Toutefois, cette variation entre les directeurs n'explique pas l'ensemble des variations de la période d'observation et ne peut expliquer les variations que l'on retrouve à l'intérieur du mandat du directeur ${ }^{6}$. Par exemple, on retrouvera en 1969, sous la direction de Szabo, $67 \%$

5. Étant donné les délais liés à la mise en place de numéros spéciaux, au processus de révision et au processus de publication, il est possible qu'il y ait un délai (lag) entre l'entrée en poste du directeur et son effet direct sur les articles publiés. Pour tester cette hypothèse, nous avons mis en place des modèles alternatifs en ajoutant des délais à l'influence du directeur. Nous avons pu constater que le modèle le mieux ajusté considère un délai de publication pour les articles empiriques de 5 numéros, alors que l'effet sur la publication d'articles quantitatifs est supérieur avec un délai de 4 numéros; un délai d'environ 2 ans.

6. Les résultats présentés dans le texte sont extraits de modèles de régression logistique multivariables présentés en annexe, maintenant l'effet de l'ensemble des indicateurs constant pour obtenir l'effet indépendant de chacun de ceux-ci. Pour simplifier la discussion et faciliter la lecture, nous nous en tiendrons aux statistiques de base issues de ces modèles (c.-à-d. pourcentages) dans le texte, mais nous invitons le lecteur à consulter l'Annexe en cas de besoin. À noter qu'un modèle à effet aléatoire au niveau du numéro de la revue a été testé, mais l'effet de la variance lié au numéro est négligeable et le modèle plus simple à effets fixes sera privilégié. 
d'articles présentant une forme empirique, alors qu'en 1976, toujours sous la même direction, $17 \%$ des articles utilisaient ce format. De manière similaire en 1998 et 2002, sous la direction de Landreville, la totalité des articles empiriques était de nature quantitative alors qu'en 2000 , sous sa même direction, $44 \%$ des articles utilisaient cette technique d'analyse ${ }^{7}$.

L'influence des directeurs et des collaborateurs dont ils s'entourent peut aussi s'exercer de manière indirecte, par l'entremise des thèmes qu'ils choisiront de privilégier durant son mandat. La Figure 2 présente la distribution de différents thèmes abordés dans la revue en fonction de leur type, que ceux-ci soient empiriques ou discursifs, et, lorsqu'ils sont empiriques, qu'ils usent de méthodes quantitatives ou qualitatives. Il se trouve que certains thèmes se prêtent mieux à certains types d'articles que d'autres, à certaines formes de recherche que d'autres. Ainsi, les tribunaux, les prisons et les corps policiers suscitent davantage d'articles réflexifs $(61 \%)$ que les objets traditionnels d'étude de la criminologie comme les délinquants adultes, juvéniles ou la criminalité (45\%). Lorsque les organes de contrôle de la criminalité suscitent des articles empiriques, on y trouve davantage d'enquêtes qualitatives (54\%) que dans les études consacrées à la délinquance (26\%). À noter que les articles qui abordent d'autres thématiques (les femmes, les victimes ou l'intervention) présentent autant d'articles empiriques (41\%) que discursifs (59\%), et autant d'enquêtes quantitatives (55\%) que qualitatives (45\%).

Ceci étant dit, les thèmes abordés dans la revue ont assez peu changé au fil du temps. On n'observe pas de baisse ou de hausse marquée des études consacrées aux délinquants ou à la criminalité (rhô $=-0,02$; $p=0,521)$ ou des articles consacrés aux agences de contrôle (rhô $=$ $0,06 ; p=0,069)$. On note bien quelques changements, mais ils sont circonscrits: une diminution des articles consacrés aux crimes et à la criminalité $(r h \hat{o}=-0,09 ; p=0,011)$ et aux prisons ( $r h \hat{o}=-0,08$; $p=0,027)$, ainsi qu'une augmentation des articles consacrés à la police et à la sécurité privée $(r h \hat{o}=0,17 ; p<0,001)^{8}$. Cette dernière augmentation peut être attribuable en partie à l'arrivée du baccalauréat en sécurité et études policières à l'Université de Montréal en 1999.

7. La période d'observation n'aura pas d'effet significatif en contrôlant pour les autres indicateurs, mis à part pour une diminution des articles quantitatifs à la période 5 (Annexe, Modèle 6).

8. Ces résultats ont été confirmés avec des tests post-hoc vérifiant l'absence de relations non linéaires. 


\section{FIG URE 2}

Distribution des thèmes abordés dans les articles en fonction du type d'article avec intervalle de confiance (95\%)

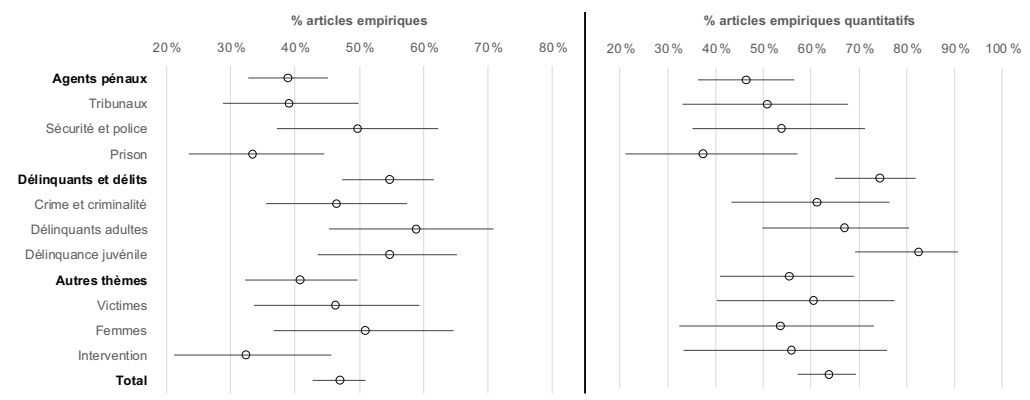

Note: Les pourcentages présentés dans cette figure sont extraits des modèles de régression logistique présentés en Annexe (Modèles 2, 3, 6, 7), contrôlant pour les autres indicateurs.

\section{Les innovations éditoriales}

Il est donc difficile de prétendre que les directeurs aient influencé le discours criminologique de la revue de manière importante en privilégiant certains sujets ou en favorisant un type d'article particulier. Il est plus vraisemblable de penser que c'est par la mise en place de politiques et de stratégies de gestion éditoriale que les directeurs ont pu influencer l'évolution du discours criminologique de la revue.

Les articles hors thèmes des numéros de la revue, mis en place en 1989 par Brochu, offrent une plateforme particulièrement intéressante pour les articles empiriques et les articles empiriques quantitatifs. La section hors thème fait davantage de place à la demande d'espace de publication faite par les auteurs qui échappent ainsi au contrôle exercé par les thématiques imposées par les comités de rédaction. On observe notamment que les études empiriques sont beaucoup plus nombreuses dans la section hors thème que dans la section thématique (72\% vs $38 \%)$. De manière similaire, la section hors thème présente plus d'articles empiriques quantitatifs $(75 \%)$ en comparaison de la section thématique $(55 \%)^{9}$.

9. Voir Annexe, Modèles 3 et 7. 
De fait, la proportion d'articles empiriques dans la section thématique est relativement stable au cours des trois périodes (Période 3 : $44 \%$; Période 4: $42 \%$; Période 5: $49 \%)^{10}$. On comprend que dans la section thématique, les comités de rédaction souhaitent équilibrer la proportion relative d'articles réflexifs qui proposent un bilan des questions et des connaissances sur un sujet et d'articles empiriques consacrés à ce thème. Toutefois, l'offre d'espace de publication pour les articles empiriques quantitatifs pour ces sections thématiques a diminué durant la dernière décennie (Période 3: 81\%; Période 4: 82\%; Période 5: $37 \%)$. En revanche, les articles empiriques sont devenus pratiquement la norme dans la section hors thème au cours des dernières années (Période 3: 52\%; Période 4: 74\%; Période 5: $92 \%$ ), augmentant ainsi les possibilités de publication pour ce type d'article. Toutefois, l'augmentation de l'offre de publications empiriques dans les sections hors thème a été comblée par l'augmentation de la demande d'articles utilisant une méthode qualitative (Période 3: 8\%; Période 4: $27 \%$; Période 5 : $35 \%$ ). Cette observation témoigne de la volonté, de plus en plus apparente avec le temps, d'arriver à un équilibre entre les différents types d'articles: discursifs, qualitatifs, quantitatifs.

Il devient également manifeste que le discours criminologique proposé par la revue Criminologie ne se limite plus à celui des auteurs qui sont affiliés à l'Université de Montréal: leur proportion qui était de $76 \%$ avant 1986 chute à $38 \%$ par la suite. Cette diversification s'opère à la fois dans les articles empiriques (79\% vs 44\%) et dans le sous-ensemble des articles empiriques quantitatifs (81\% vs $51 \%$ ). Le nombre d'auteurs affiliés à des universités situées dans d'autres pays augmente aussi de manière appréciable (10\% vs $27 \%)$. En diversifiant la provenance d'affiliation des auteurs, les directeurs offrent à leurs lecteurs une appréciation plus juste de la diversité de leurs perspectives et de leurs intérêts. La sollicitation d'auteurs étrangers a contribué, partiellement, à moduler le statut de la recherche empirique dans le discours criminologique qu'offre la revue: ils publient moins d'articles empiriques que les auteurs de l'Université de Montréal (42\% vs 52\%) et, ceux qui en publient, privilégient davantage les méthodes qualitatives $(51 \% \text { vs } 39 \%)^{11}$.

10. Ces résultats proviennent d'un modèle n'incluant que les périodes suivant l'arrivée de la section hors thème qui intègre un effet d'interaction entre la période et le fait qu'un article fasse partie de cette section (Annexe, Modèles 4 et 8 ).

11. Voir Annexe, Modèles 3 et 7. 


\section{S'assurer d'un contrôle de qualité}

En ouvrant la section hors thème, Serge Brochu a imposé un contrôle éditorial moins envahissant sur les thèmes des manuscrits que les auteurs pouvaient soumettre à la revue. Cette liberté accrue s'est cependant accompagnée de normes de publication plus uniformes. Ainsi, le nombre de pages imposé aux articles ne diffère maintenant guère d'un auteur à l'autre, et ce, depuis 1987. Durant la période des débuts (19681974), la longueur moyenne d'un article est de 54 pages et la variation autour de cette moyenne considérable puisqu'elle était de $62 \%$ $(\sigma=33,6)$. Durant la période de transition (de 1975 à 1986), les articles sont presque trop courts (16 pages en moyenne), mais la variation autour de la moyenne continue d'être substantielle $\left(c_{v}=54 \% ; \sigma=8,4\right)$. Avant 1987, on trouvait aussi bien des rapports de recherche de plus de 100 pages que des notes de lecture ou des commentaires plutôt anecdotiques (quoique distrayants). Ce n'est qu'après 1986 que l'espace accordé à chaque auteur devient uniforme, avec 21 pages en moyenne et un coefficient restreint de variation de $34 \%(\sigma=7,2)$.

En ce qui concerne spécifiquement les articles empiriques utilisant une méthode quantitative, le contrôle de la qualité sera assuré, en partie, par la complexité des analyses présentées. La Figure 3 présente l'évolution de la complexité des analyses publiées dans les articles de la revue Criminologie au fil du temps ${ }^{12}$. Les exigences en matière de recherches empiriques quantitatives étaient minimales avant 1987. Les articles descriptifs étaient dominants entre 1975 et 1986. Ils diminuent progressivement durant les années 1990. La proportion d'articles de complexité intermédiaire est constante tout au long de la période. Les analyses plus complexes, en revanche, augmentent de manière appréciable sous la direction de Pierre Landreville et de Dianne Casoni. On

12. La Figure 2 présente la proportion d'articles empiriques utilisant une méthode quantitative en fonction du type d'analyse réalisée estimée à l'aide d'une équation de régression logistique prenant l'année de publication comme prédicteur. Pour observer les tendances temporelles non linéaires, les fonctions polynomiales de l'année ont été extraites. Pour la prédiction de la tendance des analyses univariées, la fonction d'ordre trois permettait un meilleur ajustement, pour la prédiction de la tendance des analyses bivariées, on n'observe pas de fluctuations dans le temps, alors que pour prédire la tendance des articles utilisant des analyses multivariables, la fonction d'ordre trois fut privilégiée (Annexe, Modèles $9,10,11)$. À noter qu'une équation incluant d'autres indicateurs (c.-à-d. les thèmes, la section hors thème, la provenance de l'auteur, les directeurs) n'augmentait pas l'ajustement du modèle (AICc analyses univariées $=214,2$ vs 212,2; bivariées $=238,4$ vs 227,2 ; multivariables $=237,2$ vs 231,9). Le modèle le plus simple n'incluant que la variation dans le temps sera présenté. 
y trouve une proportion qui n'est pas sans rappeler celle des années d'Acta Criminologica: mais ces articles, présentant des analyses déductives plutôt que des modèles inductifs ou exploratoires, s'adressent à un public averti sans doute beaucoup plus large.

F I G URE 3

Proportion d'articles empiriques quantitatifs en fonction de la complexité des analyses

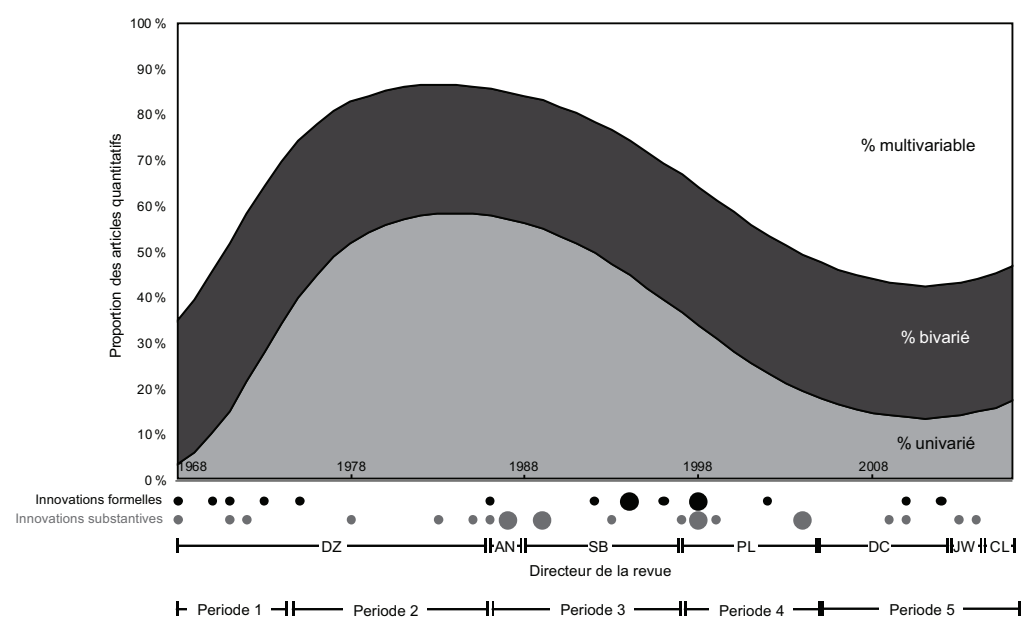

$\mathrm{DZ}$ : Denis Szabo; AN : André Normandeau; SB: Serge Brochu; PL : Pierre Landreville;

Note: Les courbes de tendance présentées dans cette figure sont extraites des modèles de régression logistique présentés en Annexe (Modèles 9, 10, 11).

Finalement, une manière complémentaire d'évaluer la hausse des standards est d'évaluer l'ouverture des directeurs, et de leurs comités de rédaction, aux recherches quantitatives innovatrices. On peut observer au bas de la Figure 3 la distribution chronologique des innovations, substantives (c.-à-d. données et devis) et formelles (c.-à-d. modèles statistiques), qui seront présentées dans cette section.

Première période: 1968-1974

Les enquêtes de délinquance autorévélée ont été introduites dans la revue en 1971 par Le Blanc, pour mettre en perspective la réaction sociale en fonction de la classe sociale chez un échantillon de jeunes. Akman, Normandeau, Sellin et Wolfgang (1968) évaluèrent, dans le 


\section{F I G RE 4}

Schématisation d'un groupe de distribution de drogue

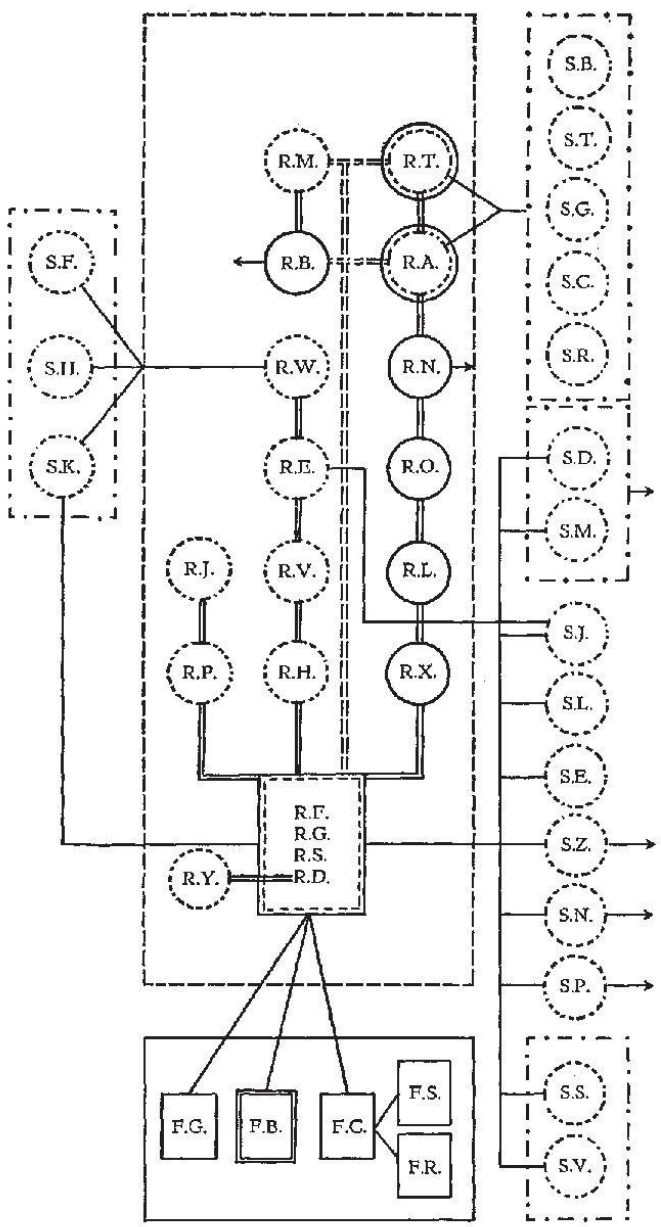

FIG. 3 : La structure du groupe,

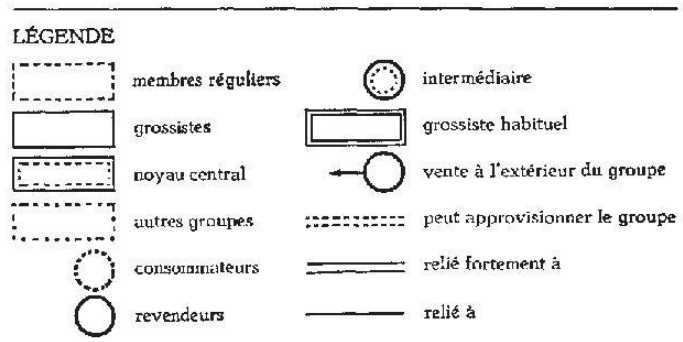


contexte canadien, la gravité des délits en sondant différentes populations et en utilisant la méthodologie psychométrique des échelles d'amplitude. Dès les débuts de la revue, Le Blanc (1972) propose une analyse sociométrique des réseaux criminels, en l'occurrence celui d'un groupe de distribution de drogues illicites (Figure 4).

Les innovations formelles ont la cote dans les débuts de la revue (Acta Criminologica). Elles prennent la forme d'analyses multivariables qui offrent des techniques empiriques pour effectuer des typologies ou pour déterminer les dimensions d'un concept général en dimensions ou facteurs par analyse factorielle. Dès le premier numéro de la revue, Szabo, Le Blanc, Deslauriers et Gagné (1968) ont observé les dimensions qui sous-tendent l'attitude morale chez les jeunes: l'autorité, le conformisme, l'aspiration, l'anxiété hédoniste et l'autovalorisation. Robert et Faugeron (1973) proposent une typologie des représentations que se font les citoyens du système de justice criminelle. Shoham, Rahav et Kreizler (1970) présentèrent la première analyse de fonction discriminante dans la revue Criminologie, présentant les facteurs permettant de distinguer les jeunes délinquants des jeunes dits conformistes. Marc Le Blanc (1971) utilise la technique des arbres décisionnels pour comprendre l'influence des caractéristiques des jeunes délinquants sur leur évolution dans le système de justice.

\section{Deuxième période: 1975-1986}

Cette période fut pauvre tant en innovations substantives que formelles. Néanmoins, la première étude dans Criminologie à sonder la perspective de la victime, fut publiée pendant cette période par Baril et Morrissette (1985), qui s'intéressèrent à la réaction des commerçants de Montréal quant à la victimisation par vol qualifié. Durand (1983) introduit aussi la notion de carte mentale subjective qui lui permet de donner un cadre géographique aux zones urbaines qui suscitent un sentiment de peur et aux raisons de cette insécurité. Les devis de recherche expérimentaux ou quasi expérimentaux constituent en eux-mêmes une forme particulière d'innovation substantive dans la littérature criminologique. Brill (1978) présente un devis de ce type, comparant le succès d'un traitement auprès de délinquants juvéniles en fonction du niveau d'organisation de leur résidence. Les recensions des écrits systématiques ont aussi fait l'objet d'innovation lorsqu'on s'est avisé que l'on pouvait agréger, par méta-analyse, les résultats obtenus par les enquêtes empiriques quanti- 
tatives méthodologiquement comparables. Loeber et Stouthamer-Loeber (1986) ont ainsi évalué les prédicteurs comportementaux et circonstanciels de la délinquance selon l'ensemble des études publiées sur le sujet. Les innovations formelles se font rares. Tout de même, en 1975, Le Blanc publia dans Criminologie la première analyse de prédiction de tendance de la délinquance juvénile au Québec à l'aide du modèle de prédiction de Box et Jenkins (1970), un modèle de base encore utilisé aujourd'hui pour la prédiction de séries chronologiques. Le Blanc (1986) fut aussi le premier à utiliser les modèles de régression multiple, présentant un modèle explicatif intégré des comportements délinquants.

\section{Troisième période: 1987-1996}

La troisième période de la revue a été, heureusement, particulièrement riche en innovations de toutes sortes. Les médias ont offert aux auteurs de la revue de nouvelles possibilités de recherche. Gabor et Weimann (1987) furent les premiers dans Criminologie à utiliser ces données pour observer la représentation de la délinquance dans les médias. Dans le même numéro, Los (1987) a analysé le contenu et le discours utilisés dans des articles de journaux pour étudier la perception véhiculée par les médias écrits sur la nouvelle Loi sur les jeunes contrevenants. Notons aussi l'utilisation ingénieuse des petites annonces par Leguerrier (1989), dans le but d'évaluer l'évolution du marché des entreprises de prostitution commerciale à Montréal. Pour sa part, Cordeau (1989) a utilisé le journal Allo Police pour offrir une reconstitution très détaillée de la dynamique des règlements de compte dans le milieu criminel montréalais. Pour leur part, Jacob, McKibben et Proulx (1993) présentèrent une innovation en termes de mesure, évaluant la déviance sexuelle d'adolescents accusés de délinquance sexuelle utilisant la pléthysmographie pénienne. Un devis quasi expérimental a également été utilisé pour évaluer l'impact des caméras de surveillance dans les lieux publics (Grandmaison et Tremblay, 1997).

Du côté des innovations formelles, les analyses de fonction discriminante furent graduellement remplacées par la régression logistique, plus flexible et plus robuste (Press et Wilson, 1978). Pellerin et al. (1996) furent les premiers à utiliser ces modèles dans Criminologie pour évaluer les caractéristiques individuelles qui influencent la récidive auprès des agresseurs sexuels. Dans une analyse géographique de la criminalité, Élie et Legendre (1992) ont mesuré, dans un quartier de 
Montréal, l'évolution des délits répertoriés de manière géographique, mais aussi de manière temporelle (Figure 5). Ils ont ainsi mesuré un concept important en géocriminologie, la mobilité des délinquants. La revue Criminologie publia d'ailleurs un numéro entier sur les modèles formels concernant la criminologie spatiale en 1994 (Ouimet, 1994). Notons en particulier l'analyse originale de Brantingham et Brantingham (1994) sur la concentration géographique locale de la criminalité.

\section{FIGURE 5}

Cartographie des valeurs prédites par un modèle de régression spatiale des introductions par effraction

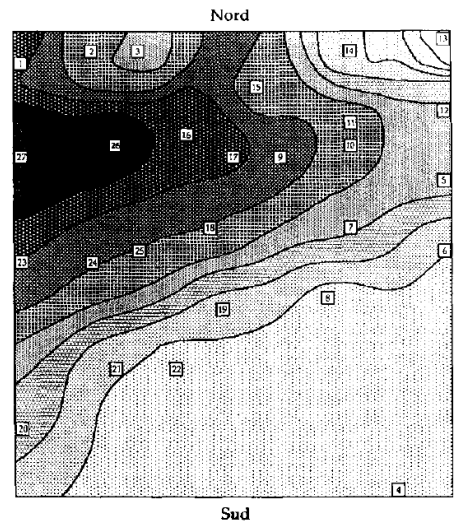

Introductions par effraction

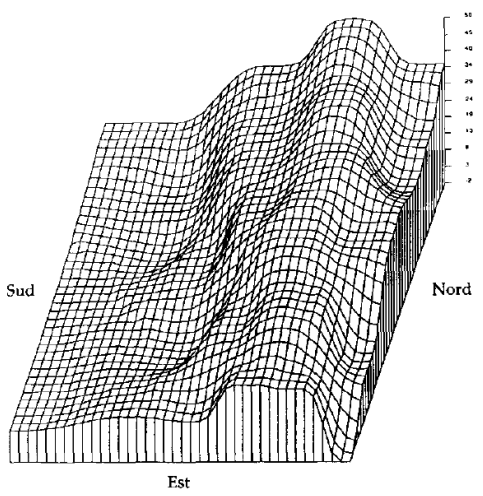

Introductions par effraction

Source: Élie et Legendre, 1992

Quatrième période: 1997-2004

Les enquêtes de délinquance autorévélée ont été enrichies lorsqu'on s'est avisé que les délinquants interrogés pouvaient nous renseigner très précisément sur leur réseau social de pairs délinquants, leur capital social ou encore les revenus obtenus par ces délits, c'est-à-dire leur performance criminelle (Charest, 2004; Morselli et Tremblay, 2004; Robitaille, 2004). Du côté des devis de recherche, Aebi, Ribeaud et Killias (1999) combinèrent enquête de délinquance autorévélée et recherche évaluative pour examiner l'effet d'un programme de réduction des méfaits auprès d'une population de consommateurs d'héroïne. Vitaro et Tremblay (1998) ont également fait une étude à assignation aléatoire pour évaluer un programme de prévention de l'agressivité chez 
des enfants en bas âge. Par ailleurs, soulignons dans cet article l'originalité de l'opérationnalisation de l'agressivité des auteurs qui, s'inspirant des travaux de Pekarik, Prinz, Liebert, Weintraub et Neale (1976), est basée sur les jugements portés par les pairs des sujets et mesure l'agressivité vécue et perçue par l'entourage.

Sur le plan des innovations formelles, Degailler (1998), dans une étude sur l'évolution de la sécurité privée au Québec, a été le premier auteur de la revue à présenter une analyse de tendance qui, en utilisant des paramètres non linéaires, permet de prédire une courbure temporelle. Ouimet et Blais (2002) présentèrent par la suite une analyse multivariable complète avec terme autorégressif, prédisant l'évolution de la criminalité en se basant sur les caractéristiques sociodémographiques de la population. Les modèles multiniveaux (aussi appelés modèles mixtes, modèles hiérarchiques ou modèles à effet aléatoire) sont de plus en plus utilisés pour prendre en considération les dimensions multiples et entrecroisées des devis et des unités d'analyse. Haggerty, Catalano, Harachi et Abbott (1998) furent les premiers à utiliser ce type de modèle dans la revue Criminologie pour décrire l'implantation d'un programme de prévention des problèmes de comportement à l'adolescence.

\section{Cinquième période: 2005-2016}

Deux analyses de réseaux sociocentriques sont publiées durant cette période: celle de Descormiers et Morselli (2010) combine des entrevues de groupes auprès de délinquants juvéniles et une analyse sociocentrique pour étudier la structure des conflits et des alliances entre les gangs de rue à Montréal; et celle de Savoie-Gargiso et Morselli (2013) exploite la richesse des archives d'enquêtes policières pour étudier la structure d'un réseau de prostitution. À noter que ces recherches sont mixtes, en partie qualitatives, en partie quantitatives. Boivin, Lamige et Tremblay (2009) ont aussi utilisé un devis de recherche quasi expérimental pour constater l'impact d'une frappe policière sur un immeuble malfamé de Montréal. Même si les sondages de victimisation ont été introduits en 1988 au Canada, ce n'est qu'en 2014 qu'ils ont retenu l'attention d'auteurs de la revue (Lebeau, Wemmers, Cyr et Chamberland, 2014).

Du côté des innovations formelles, Ouellet (2012) procède à une analyse multiniveau des séquences de rechute des délinquants et des joueurs compulsifs sur la base de questionnaires de délinquance révélée 
qui incorporent un calendrier détaillé des facteurs dynamiques de leurs trajectoires de vie. Combinant les méthodes de classification et les trajectoires de délinquance, Lussier (2010) s'inspire des travaux de Nagin et Land (1993) et proposera une typologie des trajectoires de récidive (Figure 6).

\section{FIGURE 6}

\section{Représentation graphique des trajectoires criminelles violentes et sexuelles d'un échantillon de délinquants sexuels}

Trajectoires de comportements violents/sexuels

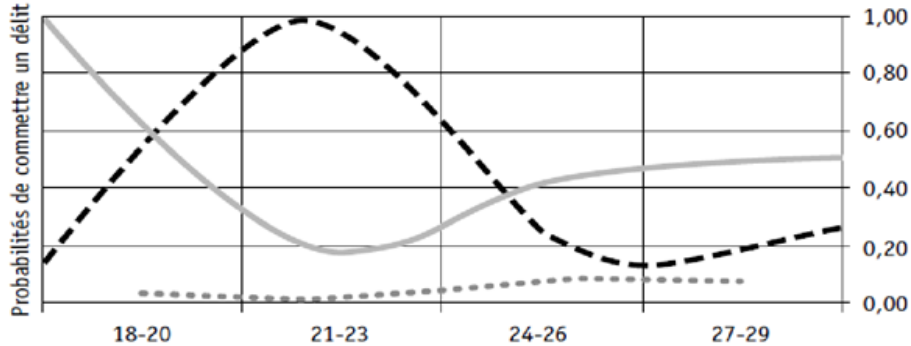

Âge du délinquant au moment de l'arrestation

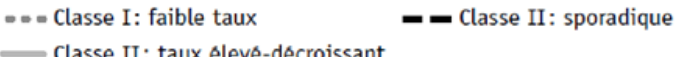

Source: Lussier, 2010

Les années des débuts, Acta Criminologica, sont atypiques et deviennent difficiles à comparer aux autres périodes en termes d'innovation. On y retrouve très peu d'articles quantitatifs $(n=9)$ et ceux-ci sont principalement publiés par des auteurs du département de l'époque, constitué d'une maîtrise seulement, donc principalement axé sur la recherche. Il est d'ailleurs à noter que sur l'ensemble des innovations de la période $(n=7)$, plus de la moitié sont propulsées par Marc Le Blanc $(n=4)$. Il n'en reste pas moins que le taux d'innovation de cette époque est impressionnant (74\%). Les articles innovants ont été rares entre 1974 et 1984: sur 21 articles empiriques quantitatifs publiés, nous n'avons observé que 3 innovations majeures (14\%). S'ensuivirent deux grandes périodes productives en termes d'innovation, avec Serge Brochu et Pierre Landreville à la direction, le taux d'innovation est monté à $21 \%$ dans chacune des périodes. On observe toutefois un essoufflement depuis 2005 : seulement 6 études se démarquent sur une masse impor- 
tante d'études quantitatives publiées $(n=58)$, avec un taux d'innovation de $10 \%$. Il est difficile de statuer sur ce déclin. Le décompte n'incorpore pas les travaux qualitatifs ou discursifs qui se sont démarqués. Il est possible aussi que nos critères d'innovation soient contaminés par un biais de saturation: les articles innovants des cohortes antérieures diminuent les opportunités d'innovation des cohortes suivantes. Mais on ne peut exclure non plus que le désir de diversifier la provenance des affiliations universitaires, d'équilibrer la part relative des enquêtes empiriques qualitatives et quantitatives, ou l'arrivée de nouvelles priorités (la professionnalisation des criminologues, le développement des études en études policières) aient relégué au second plan la demande d'études qui s'écartent des sentiers battus.

\section{Conclusion}

La revue Criminologie n'existerait pas si elle n'avait pas bénéficié d'un contexte favorable à son émergence, en l'occurrence son rattachement à une discipline universitaire reconnue et un lectorat de professionnels identifiés à cette discipline (Poupart, 2004). Il se trouve cependant que la trajectoire de la revue à ses débuts et jusqu'en 1986 a été erratique et que, n'eût été la reprise en main par une nouvelle génération de directeurs (et des comités de rédaction dont ils s'entourent), la revue Criminologie aurait bien pu disparaitre. Les innovations éditoriales qui ont assuré la survie de la revue ont pris plusieurs formes et permettent implicitement de définir en quoi consiste un «bon directeur» de revue scientifique.

Tout d'abord, la formule thématique de la revue était importante parce qu'elle permettait d'offrir à un lectorat plus élargi une proportion à peu près équilibrée d'articles de réflexion et d'investigations concrètes sur des enjeux ou des sujets particuliers. Mais en ouvrant une section hors thème, elle a aussi accordé plus de liberté aux auteurs de soumettre à la revue des articles d'intérêt pour ses lecteurs, ce qui s'est traduit, dans les faits, par une place croissante aux études empiriques.

Parallèlement, les directeurs successifs de la revue se sont employés à diversifier et à équilibrer les caractéristiques des auteurs qu'ils publiaient: les affiliations universitaires des auteurs se sont diversifiées au fil du temps et la proportion de recherches empiriques qualitatives et quantitatives est devenue plus équilibrée. Ils se sont également employés à imposer à tous les auteurs les mêmes contraintes (par ex.: la longueur des articles publiés) et à hausser les standards de qualité des articles (analyses plus élaborées, ouverture aux innovations). 
Dans cette étude consacrée à l'historique d'une revue scientifique, il nous a semblé naturel d'adopter la perspective des directeurs de la revue (ou de leurs comités de rédaction) plutôt que des auteurs eux-mêmes ou d'une fraction d'entre eux. Il va de soi qu'un examen des stratégies de publications des auteurs aurait permis de situer la place qu'occupe la revue Criminologie par rapport à d'autres forums concurrentiels de publication, une dimension qui n'a pas été abordée. Par ailleurs, notre tentative d'évaluer la qualité des articles de la revue en procédant à un relevé de leurs innovations substantives ou formelles est préliminaire et largement exploratoire, même si nos résultats semblent corroborés par d'autres indicateurs comme le déclin des compilations statistiques simplement descriptives et l'augmentation des analyses statistiques plus élaborées publiées dans la revue ${ }^{13}$. Notre relevé est incomplet à plusieurs égards. Il devrait être possible de procéder à un relevé des articles réflexifs qui se démarquent par leurs innovations discursives ou à un relevé des articles empiriques qualitatifs qui se démarquent par leurs innovations substantives. Il serait souhaitable aussi d'établir si les innovations répertoriées demeurent sans lendemain ou si elles ont effectivement influencé les articles publiés par la suite dans la revue. Le degré de cohésion du discours criminologique véhiculé dans la revue pourrait aussi être évalué dans une perspective historique en analysant la configuration des chaînes de citation au cours des cinq périodes et l'importance relative que les auteurs de la revue occupent dans ces chaînes.

Mentionnons pour finir que la survie d'une revue scientifique ne va jamais de soi. Il sera intéressant d'évaluer comment des facteurs aussi différents que l'avènement des revues en libre accès ${ }^{14}$, l'incorporation des criminologues dans un ordre professionnel, la pression exercée sur

13. Il est possible de croire qu'avec l'accessibilité croissante de puissance du calcul informatique, l'augmentation de la complexité des analyses ne tire pas à sa fin. Nordhaus (2007) a montré que le rapport du coût des ordinateurs en fonction de leur puissance de calcul (mesuré par le nombre d'opérations par seconde) suit une courbe logarithmique descendante à travers les deux derniers siècles; les ordinateurs puissants devenant de plus en plus abordables. On observe d'ailleurs une corrélation significative entre ce ratio d'efficacité moyen à travers le temps et la proportion d'utilisation des modèles plus complexes dans notre série chronologique ( $r$ ho $=-0,32 ; \mathrm{p}=0,027)$.

14. L'accès aux revues scientifiques est très coûteux et reste la plupart du temps accessible seulement pour les bibliothèques universitaires. On retrouve toutefois de plus en plus de revues en accès libre, qui sont accessibles gratuitement en ligne. Par exemple, le Directory of Open Access Journal offre un moteur de recherche de revues en accès libre sur des thématiques variées. Ne reste qu'aux chercheurs à participer à la floraison de ces revues. 
les chercheurs par les organismes subventionnaires de recherche, quant au partage de leurs banques de données, mais aussi les initiatives des chercheurs eux-mêmes à partager leurs données (Allnutt et Goulet, $2015)^{15}$, ou encore l'avènement du big data ${ }^{16}$, influenceront la configuration des études qui définissent le discours criminologique de la revue Criminologie dans les années à venir.

\section{Références}

Aebi, M., Ribeaud, D. et Killias, M. (1999). Prescription médicale de stupéfiants et délinquance: Résultats des essais suisses. Criminologie, 32(2), 127-148.

Akman, D., Normandeau, A., Sellin, T. et Wolfgang, M. (1968). Towards the measurement of criminality in Canada. Acta Criminologica, 1(1), 135-260.

Allnutt, V. et Goulet, M.-A. (2015). Libre accès: vers une science plus ouverte. Documentation et bibliotbèques, 61(1), 3-5.

Baril, M. et Morrissette, A. (1985). Du côté des victimes, une autre perspective sur le vol à main armée. Criminologie, 18(2), 117-133.

Boivin, R., Lamige, C. et Tremblay, P. (2009). La police devrait-elle cibler les taudis malfamés? Criminologie, 42(1), 225-245.

Box, G. et Jenkins, G. (1970). Time series analysis: Forecasting and control. San Francisco, CA: Holden-Day.

Brantingham, P. et Brantingham, P. (1994). La concentration spatiale relative de la criminalité et son analyse: vers un renouvellement de la criminologie environnementale. Criminologie, 27(1), 81-97.

Brill, R. (1978). Traitement des jeunes délinquants: modèle d'appariement selon le niveau conceptuel. Criminologie, 11(1), 46-64.

Charest, M. (2004). Peut-on se fier aux délinquants pour estimer leurs gains criminels? Criminologie, 37(2), 63-87.

Charette, Y. et Papachristos, A. (2017). The network dynamics of co-offending careers. Social Networks, 51, 3-13.

Cordeau, G. (1989). Les homicides entre délinquants: une analyse des conflits qui provoquent des règlements de comptes. Criminologie, 22(2), 13-34.

Degailler, F. (1998). Sécurité privée au Québec, un marché en évolution? Criminologie, 31(2), 47-67.

15. Soulignons l'existence de certaines initiatives comme l'Interuniversity Consortium for Political and Social Research qui offre une plateforme en ligne aux chercheurs pour partager leurs données et leur permettre d'avoir accès à plus de 65000 bases de données, sans frais, ou encore le projet Zenodo qui permet aussi aux chercheurs de partager leurs données.

16. Il est maintenant possible d'avoir accès, à la minute près, à tous les délits qui sont rapportés aux services de police, avec la géolocalisation de manière précise, sur une période de quelques décennies (Charette et Papachristos, 2017; Hipp et Kim, 2016; Song, Spicer, Brantingham et Frank, 2013). 
Descormiers, K. et Morselli, C. (2010). Analyse de la structure sociale des conflits et des alliances intergangs. Criminologie, 43(1), 57-89.

Durand, S. (1983). La peur du crime dans un quartier de Montréal: Côte-desNeiges. Criminologie, 16(1), 85-99.

Élie, D. et Legendre, P. (1992). Autocorrélation spatiale et déplacement de la criminalité. Criminologie, 25(2), 139-154.

Gabor, T. et Weimann, G. (1987). La couverture du crime par la presse: un portait fidèle ou déformé? Criminologie, 20(1), 79-98.

Grandmaison, R. et Tremblay, P. (1997). Évaluation des effets de la télé-surveillance sur la criminalité commise dans 13 stations du métro de Montréal. Criminologie, 30(1), 93-110.

Haggerty, K., Catalano, R., Harachi, T. et Abbott, B. (1998). Description de l'implantation d'un programme de prévention des problèmes de comportement à l'adolescence. Criminologie, 31(1), 25-47.

Hidalgo, B. et Goodman, M. (2013). Multivariate or multivariable regression? American Journal of Public Health, 103(1), 39-40.

Hipp, J. R. et Kim, Y.-A. (2016). Measuring crime concentration across cities of varying size: Complications based on the spatial and temporal scale employed. Journal of quantitative criminology, 33(3), 595-632.

Jacob, M., McKibben, A. et Proulx, J. (1993). Étude descriptive et comparative d'une population d'adolescents agresseurs sexuels. Criminologie, 26(1), 133-163.

Le Blanc, M. (1971). La réaction sociale à la délinquance juvénile: Une analyse stigmatique. Acta Criminologica, 4(1), 113-191.

Le Blanc, M. (1972). La délinquance en milieu aisé: Un groupe culture d'adeptes de la drogue. Acta Criminologica, 5(1), 107-181.

Le Blanc, M. (1975). La délinquance d'hier et de demain au Québec. Criminologie, 8(1-2), 145-158.

Le Blanc, M. (1986). Pour une approche intégrative de la conduite délinquante des adolescents. Criminologie, 19(1), 73-95.

Lebeau, A., Wemmers, J.-A., Cyr, K. et Chamberland, C. (2014). Comparaison de deux enquêtes de victimisation. Criminologie, 47(1), 105-126.

Leguerrier, Y. (1989). Les entreprises de prostitution commerciale: les commerces éphémères des marchés illicites. Criminologie, 22(2), 35-63.

Loeber, R. et Stouthamer-Loeber, M. (1986). La prédiction de la délinquance. Criminologie, 19(2), 49-77.

Los, M. (1987). La loi sur les jeunes contrevenants et les masse-médias. Criminologie, 20(1), 7-33.

Lussier, P. (2010). Trajectoires criminelles et récidive des délinquants sexuels adultes: l'hypothèse «statique» revue et corrigée. Criminologie, 43(2), 269-302.

Morselli, C. et Tremblay, P. (2004). Délinquance, performance et capital social: une théorie sociologique des carrières criminelles1. Criminologie, 37(2), 89-122.

Nagin, D. S. et Land, K. (1993). Age, criminal careers, and population heterogeneity: Specification and estimation of a nonparametric, mixed Poisson model. Criminology, 31(3), 327-362. 
Nordhaus, W. D. (2007). Two centuries of productivity growth in computing. Journal of economic history, 67(1), 128-159.

Ouellet, F. (2012). La dynamique des séquences de rechute chez des joueurs excessifs et des joueurs délinquants. Criminologie, 45(2), 51-85.

Ouimet, M. (dir.). (1994). Analyse spatiale du crime [Numéro spécial]. Criminologie, 27(1).

Ouimet, M. et Blais, É. (2002). L'impact de la démographie sur les tendances de la criminalité au Québec de 1962 à 1999. Criminologie, 35(1), 5-23.

Pekarik, E. G., Prinz, R. J., Liebert, D. E., Weintraub, D. et Neale, J. M. (1976). The pupil evaluation inventory: A sociometric technique for assessing children's social behavior. Journal of abnormal child psychology, 4(1), 83-97.

Pellerin, B., Proulx, J., Ouimet, M., Paradis, Y., McKibben, A. et Aubut, J. (1996). Étude de la récidive post-traitement chez des agresseurs sexuels judiciarisés. Criminologie, 29(1), 85-108.

Poupart, J. (2004). L'institutionnalisation de la criminologie au Québec: une lecture sociohistorique. Criminologie, 37(1), 71-105.

Press, J. S. et Wilson, S. (1978). Choosing between logistic regression and discriminant analysis. Journal of the American Statistical Association, 73(364), 699-705.

R Development Core Team. (2017). $R$ : A language and environment for statistical computing. Vienne, Autriche: R Foundation for statistical computing. Repéré à http://www.R-project.org/

Robert, P. et Faugeron, C. (1973). Représentations du système de justice criminelle: Essai de typologie. Acta Criminologica, 6(1), 13-65.

Robitaille, C. (2004). À qui profite le crime? Les facteurs individuels de la réussite criminelle. Criminologie, 37(2), 33-62.

Savoie-Gargiso, I. et Morselli, C. (2013). Homme à femmes: le proxénète et sa place parmi les prostituées. Criminologie, 46(1), 243-268.

Shoham, S., Rahav, G. et Kreizler, A. (1970). The measurement of movements on the conformity-deviance continuum as an auxiliary tool for actionresearch. Acta Criminologica, 3(1), 103-141.

Song, J., Spicer, V., Brantingham, P. et Frank, R. (2013). Crime ridges : Exploring the relationship between crime attractors and offender movement. 2013 European Intelligence and Security Informatics Conference, 75-82. doi : 10.1109/ EISIC.2013.18

Szabo, D., Le Blanc, M., Deslauriers, L. et Gagné, D. (1968). Interprétations psycho-culturelles de l'inadaptation juvénile dans la société de masse contemporaine. Acta Criminologica, 1(1), 9-133.

Vitaro, F. et Tremblay, R. (1998). Prévention de la délinquance: le rôle médiateur des pairs. Criminologie, 31(1), 49-66. 


\section{Empirical quantitative research trajectory in Criminologie journal (1968-2016) : An editorial perspective}

ABSTRACT • Each issue of a scientific journal is the result of many rounds of negotiation, beginning with the editorial board, moving to those who have agreed to help with the issue, and ending with the process of review and revision of those manuscripts that will finally be published. Journal editors must maintain a balance between theoretical and experimental articles and between those that deal with empirical research that tests a particular hypothesis and those that are exploratory and open-ended. Based on the articles published in Criminologie from 1968 to 2016 ( $\mathrm{n}=658)$, this study analyses the effect of editorial strategies on the relative share of empirical quantitative research. Analyses show that editors, through the editorial board, can influence the supply of such articles through editorial innovations (such as thematic issues, biannual publication, open topic issues, encouraging diversity in authors' locations) but can also affect submissions through an increase in standards (uniform length, complex analysis) and by soliciting innovative articles. These editorial innovations have led to a journal that is scientifically diverse and have ensured the journal's survival for half a century.

KEYWORDS - Criminologie, quantitative methods, innovations, editorial process, historical analysis.

\section{La trayectoria de la investigación empírica cuantitativa en la revista Criminologie (1968- 2016) : Una perspectiva editorial}

RESUMEN - Cada número publicado en una revista científica viene a clausurar varias rondas de negociaciones, que comienzan en el seno del comité de redacción, se desplazan luego alrededor de quienes aceptaron participar en el número, y se ramifican y se prolongan durante las evaluaciones y las revisiones de los manuscritos que serán finalmente publicados. Los sucesivos directores deben balancear la parte relativa a los artículos de reflexión y a los artículos de investigación con la proporción relativa a las investigaciones empíricas que proceden por verificación de hipótesis y aquella a las investigaciones abiertas y exploratorias. Basándose en el conjunto de los artículos publicados en la revista Criminologie de 1968 a 2016 ( $\mathrm{n}=658$ ), este estudio se interesa por el efecto de las diferentes estrategias editoriales adoptadas sobre la parte relativa que será acordada a la investigación empírica cuantitativa en el discurso criminológico. Los análisis muestran que los directores, por intermedio de los comités de redacción, pueden incidir sobre la oferta de innovaciones editoriales (introducción de números temáticos, publicación semestral, apertura hacia las publicaciones de tema libre, diversificación del origen de los autores), pero también controlar esta oferta por medio de un alza de los estándares (uniformidad del tamaño de los artículos, complejidad de los análisis) y por una solicitud activa de artículos innovadores. Estas innovaciones editoriales permitieron diversificar el discurso científico de la revista $y$, al final, aseguraron la supervivencia de la revista durante medio siglo.

Palabras Claves - Revista Criminologie, método cuantitativo, innovaciones, proceso editorial, análisis histórico. 


\section{Annexe}

Ajustement et coefficients de modèles présentés

1. Ajustement des modèles de prédiction en fonction des itérations de la fonction polynomiale du temps

\begin{tabular}{|l|c|c|c|c|c|}
\hline & \multicolumn{5}{|c|}{ Ajustement du modèle (AICc) } \\
\hline Équation & $\begin{array}{c}\text { Articles } \\
\text { empiriques }\end{array}$ & $\begin{array}{c}\text { Articles } \\
\text { quantitatifs }\end{array}$ & $\begin{array}{c}\text { Analyses } \\
\text { univariées }\end{array}$ & $\begin{array}{c}\text { Analyses } \\
\text { bivariées }\end{array}$ & $\begin{array}{c}\text { Analyses } \\
\text { multivariables }\end{array}$ \\
\hline $\mathrm{i}$ & 910,68 & 410,87 & 233,64 & $227,17^{*}$ & 248,38 \\
\hline $\mathrm{I}+\mathrm{t}$ & 892,99 & 402,05 & 225,11 & 228,90 & 23,79 \\
\hline $\mathrm{I}+\mathrm{t2}$ & 894,46 & 398,00 & 217,19 & 230,62 & 234,94 \\
\hline $\mathrm{I}+\mathrm{t3}$ & 895,45 & 397,31 & $212,00^{*}$ & 232,01 & $231,71^{*}$ \\
\hline $\mathrm{I}+\mathrm{t4}$ & 897,09 & $389,49 *$ & 213,54 & 231,93 & 232,48 \\
\hline $\mathrm{I}+\mathrm{t5}$ & $890,64^{*}$ & 390,94 & 214,49 & 233,87 & 234,47 \\
\hline
\end{tabular}

* Modèle présentant l'ajustement optimal. 


\section{Coefficients des modèles présentés}

\begin{tabular}{|c|c|c|c|c|c|c|c|c|c|c|c|c|}
\hline & \multicolumn{12}{|c|}{ Modèles de régression logistique prédisant l'occurrence d'un article empirique } \\
\hline & \multicolumn{3}{|c|}{$\begin{array}{l}\text { Modèle } 1 \\
\text { Les tendances temporelles } \\
\quad(n=658)\end{array}$} & \multicolumn{3}{|c|}{$\begin{array}{l}\text { Modèle } 2 \\
\text { Les thèmes } \\
(n=658)\end{array}$} & \multicolumn{3}{|c|}{$\begin{array}{c}\text { Modèle } 3 \\
\text { Les grands thèmes } \\
(n=658)\end{array}$} & \multicolumn{3}{|c|}{$\begin{array}{l}\text { Modèle } 4 \text { Interaction hors } \\
\text { thème* Période } \\
(n=451)\end{array}$} \\
\hline & $\operatorname{Exp}(b)$ & (IC $95 \%)$ & & $\operatorname{Exp}(b)$ & (IC $95 \%)$ & & $\operatorname{Exp}(b)$ & $(\mathrm{IC} 95 \%)$ & & $\operatorname{Exp}(b)$ & (IC $95 \%)$ & \\
\hline (Ord. à l'origine) & 7,64 & {$[0,90 ; 65,25]$} & + & 0,26 & {$[0,10 ; 0,68]$} & ** & 0,17 & {$[0,06 ; 0,48]$} & *** & 0,23 & {$[0,11 ; 0,48]$} & *** \\
\hline \multicolumn{13}{|l|}{ Fonction polynomiale } \\
\hline Année & 0,37 & {$[0,19 ; 0,73]$} & ** & - & & & - & & & - & & \\
\hline Année 2 & 1,12 & {$[1,04 ; 1,20]$} & ** & - & & & - & & & - & & \\
\hline Année 3 & 0,99 & {$[0,99 ; 1,00]$} & ** & - & & & - & & & - & & \\
\hline Année 4 & 1,00 & {$[1,00 ; 1,00]$} & ** & - & & & - & & & - & & \\
\hline Année 5 & 1,00 & {$[1,00 ; 1,00]$} & $* *$ & - & & & - & & & - & & \\
\hline \multicolumn{13}{|l|}{ Période de la revue } \\
\hline Période 1 & - & & & & f́férence & & & fférence & & - & & \\
\hline Période 2 & - & & & 0,76 & {$[0,31 ; 1,82]$} & & 0,81 & {$[0,34 ; 1,95]$} & & - & & \\
\hline Période 3 & - & & & 1,16 & {$[0,47 ; 2,87]$} & & 1,22 & {$[0,49 ; 3,02]$} & & \multicolumn{3}{|c|}{ Référence } \\
\hline Période 4 & - & & & 1,07 & {$[0,43 ; 2,69]$} & & 1,32 & {$[0,53 ; 3,31]$} & & 0,92 & {$[0,52 ; 1,65]$} & \\
\hline Période 5 & - & & & 1,70 & {$[0,71 ; 4,11]$} & & 1,98 & {$[0,82 ; 4,75]$} & & 1,21 & {$[0,74 ; 1,97]$} & \\
\hline Article hors thème & - & & & 4,32 & {$[2,46 ; 7,59]$} & $* * *$ & 4,30 & {$[2,47 ; 7,50]$} & $* * *$ & 1,41 & {$[0,55 ; 3,64]$} & \\
\hline Université de Montréal & - & & & 1,48 & {$[1,03 ; 2,12]$} & * & 1,50 & {$[1,05 ; 2,15]$} & * & 1,58 & {$[1,05 ; 2,37]$} & * \\
\hline
\end{tabular}


Modèles de régression logistique prédisant l'occurrence d'un article empirique

Thème (spécifique)

\begin{tabular}{|c|c|c|c|c|c|c|}
\hline \multicolumn{2}{|c|}{ Modèle 1} & \multicolumn{2}{|c|}{ Modèle 2} & \multicolumn{2}{|r|}{ Modèle 3} & Modèle 4 Interaction hors \\
\hline $\operatorname{Exp}(b)$ & (IC $95 \%$ ) & $\operatorname{Exp}(b)$ & (IC $95 \%)$ & $\operatorname{Exp}(b)$ & (IC $95 \%$ ) & (IC $95 \%)$ \\
\hline
\end{tabular}

Victimes

Crime

Délinquants adultes

Sécurité-Police

Tribunaux

Prison

Délinquants juvéniles

Femmes

Intervention

Thème (large)

Agents pénaux

Délinquants et délits

Autres thèmes

Interactions

Période4: horsthème

Période 5: horsthème

$$
\begin{aligned}
& - \\
& - \\
& - \\
& - \\
& - \\
& - \\
& - \\
& -
\end{aligned}
$$$$
-
$$$$
\text { - }
$$$$
\text { - }
$$$$
-
$$$$
-
$$$$
\text { - }
$$$$
-
$$

$\begin{array}{llll}1,86 & {[1,06 ; 3,26]} & * & - \\ 1,87 & {[1,06 ; 3,31]} & * & - \\ 3,08 & {[1,67 ; 5,67]} & * * * & - \\ 2,14 & {[1,12 ; 4,06]} & * & - \\ 1,38 & {[0,79 ; 2,40]} & & - \\ 1,08 & {[0,60 ; 1,94]} & & - \\ 2,60 & {[1,54 ; 4,39]} & * * * & - \\ 2,24 & {[1,19 ; 4,20]} & * & - \\ 1,03 & {[0,56 ; 1,91]} & & -\end{array}$

1,84

$[1,08 ; 3,14]$

3,49

$[2,08 ; 5,84]$

1,99

$[1,23 ; 3,23]$

$-$ $\operatorname{Exp}($ b) (IC $95 \%$ ) 


\section{2. suite}

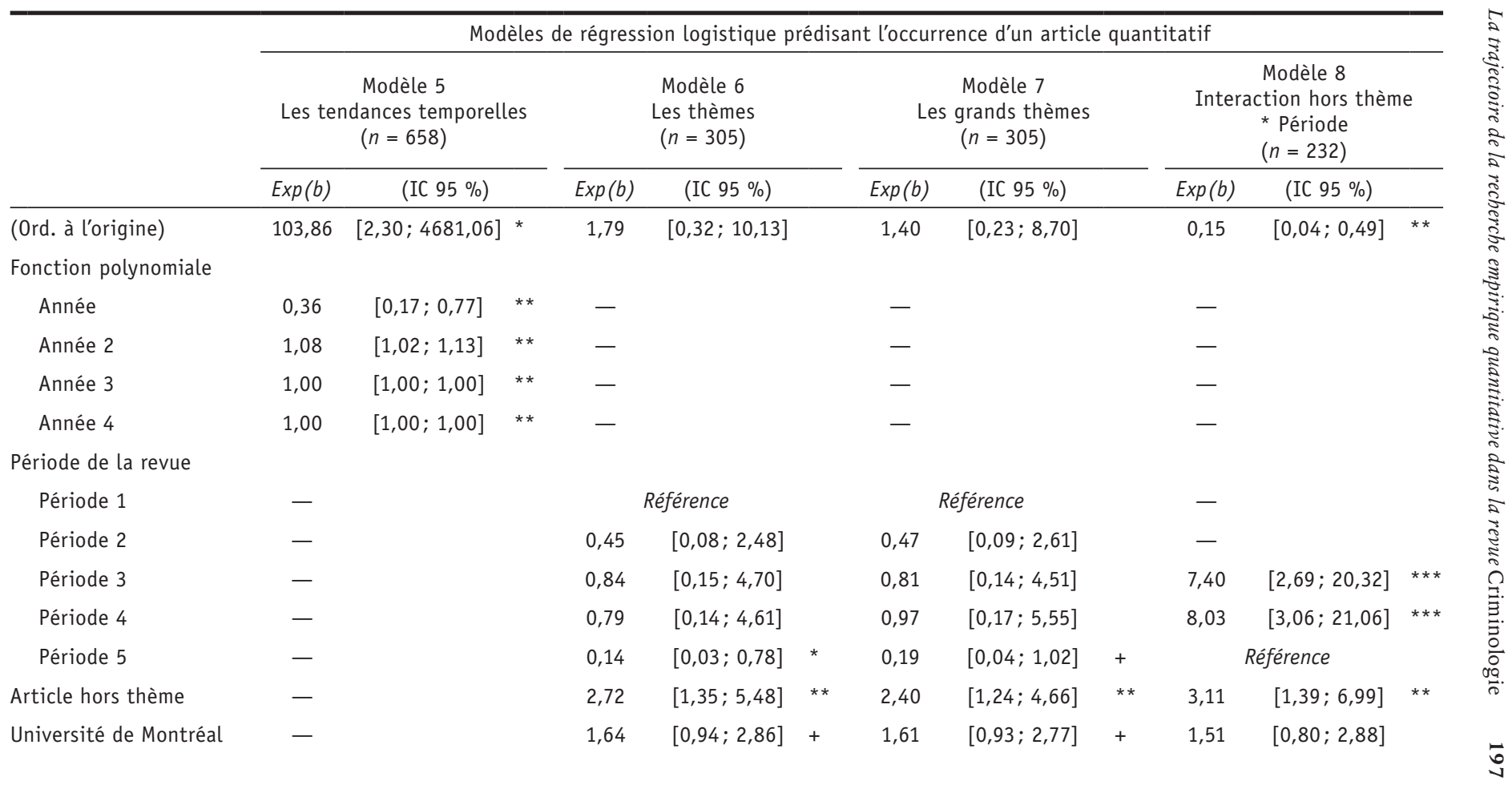




\begin{tabular}{|c|c|c|c|c|c|c|c|c|c|c|c|}
\hline & \multicolumn{11}{|c|}{ Modèles de régression logistique prédisant l'occurrence d'un article quantitatif } \\
\hline & \multicolumn{2}{|c|}{ Modèle 5} & \multicolumn{3}{|c|}{ Modèle 6} & \multicolumn{3}{|c|}{ Modèle 7} & \multicolumn{3}{|c|}{ Modèle 8} \\
\hline & $\operatorname{Exp}(b)$ & (IC $95 \%)$ & $\operatorname{Exp}(b)$ & (IC $95 \%)$ & & $\operatorname{Exp}(b)$ & (IC $95 \%)$ & & $\operatorname{Exp}(b)$ & (IC $95 \%)$ & \\
\hline \multicolumn{12}{|l|}{ Thème (spécifique) } \\
\hline Victimes & - & & 1,46 & {$[0,61 ; 3,51]$} & & - & & & - & & \\
\hline Crime & - & & 1,51 & {$[0,60 ; 3,84]$} & & - & & & - & & \\
\hline Délinquants adultes & - & & 1,94 & {$[0,76 ; 4,92]$} & & - & & & - & & \\
\hline Sécurité-Police & - & & 1,11 & {$[0,41 ; 3,01]$} & & - & & & - & & \\
\hline Tribunaux & - & & 0,98 & {$[0,37 ; 2,62]$} & & - & & & - & & \\
\hline Prison & - & & 0,57 & {$[0,21 ; 1,58]$} & & - & & & - & & \\
\hline Délinquants juvéniles & - & & 4,48 & {$[1,90 ; 10,55]$} & $* * *$ & - & & & - & & \\
\hline Femmes & - & & 1,10 & {$[0,43 ; 2,79]$} & & - & & & - & & \\
\hline Intervention & - & & 1,21 & {$[0,46 ; 3,18]$} & & - & & & - & & \\
\hline \multicolumn{12}{|l|}{ Thème (large) } \\
\hline Agents pénaux & - & & - & & & 0,93 & {$[0,40 ; 2,15]$} & & 1,34 & {$[0,46 ; 3,92]$} & \\
\hline Délinquants et délits & - & & - & & & 3,13 & {$[1,42 ; 6,88]$} & ** & 5,84 & {$[2,15 ; 15,90]$} & *** \\
\hline Autres thèmes & - & & - & & & 1,34 & {$[0,65 ; 2,76]$} & & 1,85 & {$[0,72 ; 4,71]$} & \\
\hline \multicolumn{12}{|l|}{ Interactions } \\
\hline Période3: horsthème & - & & - & & & - & & & 0,83 & {$[0,08 ; 9,14]$} & \\
\hline Période4: horsthème & - & & - & & & - & & & 0,21 & {$[0,04 ; 1,14]$} & + \\
\hline AICc & & 389,49 & & 374,13 & & & 365,57 & & & 260,86 & \\
\hline
\end{tabular}




\section{2. suite}

\begin{tabular}{|c|c|c|c|c|c|c|c|c|c|}
\hline & \multicolumn{9}{|c|}{ Complexité des analyses } \\
\hline & \multicolumn{3}{|c|}{$\begin{array}{c}\text { Modèle 9: } \\
\text { Analyses univariées } \\
(n=185)\end{array}$} & \multicolumn{3}{|c|}{$\begin{array}{c}\text { Modèle 10: } \\
\text { Analyses bivariées } \\
(n=185)\end{array}$} & \multicolumn{3}{|c|}{$\begin{array}{c}\text { Modèle 11: } \\
\text { Analyses multivariables } \\
(n=185)\end{array}$} \\
\hline & $\operatorname{Exp}(b)$ & (IC $95 \%)$ & & $\operatorname{Exp}(b)$ & (IC $95 \%)$ & & $\operatorname{Exp}(b)$ & (IC $95 \%)$ & \\
\hline (Ord. à l'origine) & 0,02 & {$[0,00 ; 0,38]$} & ** & 0,42 & {$[0,31 ; 0,58]$} & $* * *$ & 2,33 & {$[0,34 ; 15,98]$} & \\
\hline \multicolumn{10}{|c|}{ Fonction polynomiale } \\
\hline Année & 1,80 & {$[1,20 ; 2,70]$} & ** & - & & & 0,68 & {$[0,50 ; 0,92]$} & * \\
\hline Année 2 & 0,98 & {$[0,96 ; 0,99]$} & ** & - & & & 1,02 & {$[1,00 ; 1,03]$} & * \\
\hline Année 3 & 1,00 & {$[1,00 ; 1,00]$} & * & - & & & 1,00 & {$[1,00 ; 1,00]$} & * \\
\hline $\mathrm{AICC}$ & & 212,22 & & & 227,19 & & & 231,94 & \\
\hline
\end{tabular}

